\title{
Abby Day, Mia Lövheim (eds.), Modernities, Memory and Mutations. Grace Davie and the Study of Religion
}

Farnham, Ashgate, 2015, 272 p.

\section{Cornelius Crowley}

\section{CpenEdition \\ Journals}

Édition électronique

URL : http://journals.openedition.org/assr/27339

DOI : $10.4000 /$ assr.27339

ISSN : $1777-5825$

Éditeur

Éditions de l'EHESS

Édition imprimée

Date de publication : 1 octobre 2015

Pagination : 288

ISBN : 978-2-7132-2515-4

ISSN : 0335-5985

\section{Référence électronique}

Cornelius Crowley, « Abby Day, Mia Lövheim (eds.), Modernities, Memory and Mutations. Grace Davie and the Study of Religion », Archives de sciences sociales des religions [En ligne], 172 I octobredécembre, mis en ligne le 19 mai 2016, consulté le 24 septembre 2020. URL : http://

journals.openedition.org/assr/27339; DOI : https://doi.org/10.4000/assr.27339 


\section{Abby Day, Mia Lövheim (eds.), Modernities, Memory and Mutations. Grace Davie and the Study of Religion}

Farnham, Ashgate, 2015, 272 p.

Cornelius Crowley

\section{RÉFÉRENCE}

Abby Day, Mia Lövheim (eds.), Modernities, Memory and Mutations. Grace Davie and the Study of Religion, Farnham, Ashgate, 2015, 272 p. 
Ce recueil d'essais atteste l'influence exercée par Grace Davie dans les orientations, les méthodes et hypothèses, l'enracinement institutionnel et les réseaux de recherche, qui structurent actuellement l'étude du fait religieux. Si la carrière de Grace Davie, à partir d'une recherche doctorale consacrée aux courants politiques de droite dans le protestantisme français entre 1900 et 1945, s'est déroulée en Angleterre, elle a noué des collaborations durables avec des institutions en dehors de l'Angleterre (EHESS à Paris, l'université d'Uppsala en Suède) et aura coordonné plusieurs projets collaboratifs, Welfare and Religion in a European Perspective, 2003-2006, Welfare and Values in Europe, 2006-2009, le premier avec l'appui d'une fondation suédoise, le second avec un financement européen. Le compagnonnage intellectuel et la

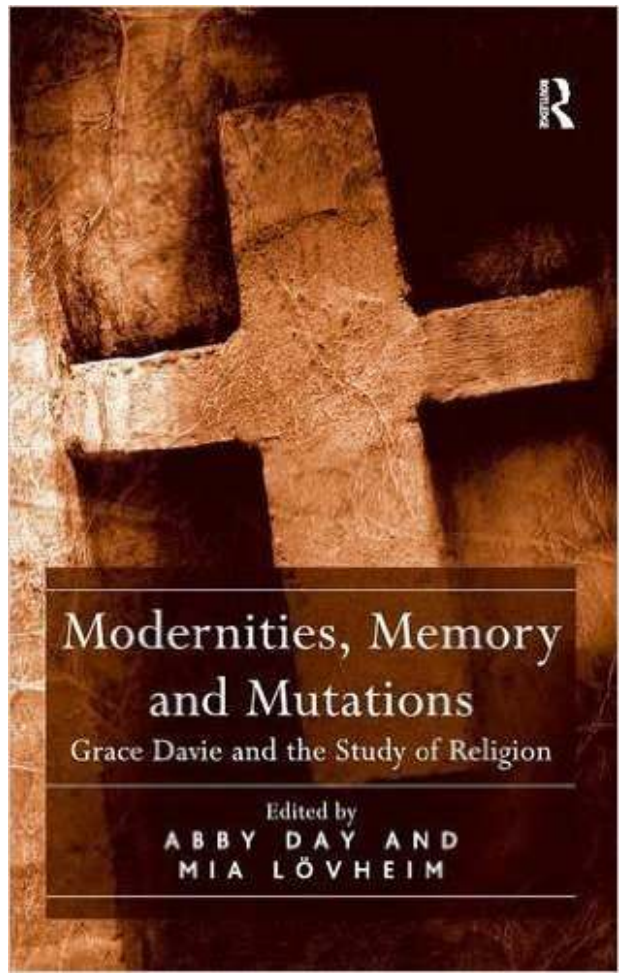
communauté d'approche développés dans ces projets se reflètent dans le rattachement institutionnel des différents participants à ce volume. Rien de plus normal, s'agissant d'un livre ayant le statut de Festschrift.

2 On peut s'interroger sur les contours de cette communauté de recherche où prennent place les contributions au présent volume: y voir le signe que la sociologie de la religion est actuellement florissante en Angleterre et dans les pays du nord-ouest de l'Europe ; ou y déceler le risque d'une clôture et d'une autovalidation de l'entreprise intellectuelle issue de ce compagnonnage. En effet, la réflexion d'Adam Possamai (p.137) à propos de la sociologie, lorsqu'elle se donne pour objet la religion, «la discipline, à force d'être par moments si autoréférentielle, ne parvenait pas à quitter des yeux les travaux prophétiques de Durkheim, Marx et Weber sur le sécularisme ", peut être retournée contre le travail collectif de ce volume, pour questionner ses présupposés, structurants et porteurs, malgré ou à cause de la sous-explicitation des thèses qui sont avancées. En effet, s'agissant des catégories mobilisées ici dans le sillage des travaux de Grace Davie, s'agissant aussi de l'inscription de ce projet collaboratif dans une opposition aussi discrète que ferme à une compréhension naguère dominante des thèses sur la modernité et la sécularisation issues de ces "travaux prophétiques », on peut postuler que le livre propose son propre mode d'autoréférentialité, d'autant plus efficace qu'elle dérive d'une entreprise collaborative, institutionnellement étayée, et que les données convoquées en appui renvoient à des faits constatés dans des enquêtes d'opinion démontrant une persistance de la référence religieuse chez les Européens (mais, à ce propos, quel crédit accorder à l'argumentation d'Anne Birgitta Pessi concernant le fait religieux en Finlande, lorsqu'elle cherche et trouve une validation empirique aux thèses de Grace Davie sur la notion de "vicarious religion ", "par procuration", dans une enquête auprès de 17 personnes interviewées, dix femmes et sept hommes?). 
3 Les différentes contributions à ce volume relèvent les signes de cette persistance de la religion, invoquée en réfutation de l'hypothèse d'une modernité par sécularisation valant «rationalisation ». D'où la fonction cruciale, dans un projet de recherche qui souscrit à une hypothèse "post-séculariste ", des critères servant à définir "belonging ", l'appartenance, et "believing", la croyance. D'où l'importance de l'articulation indiscutablement interactive qui existe entre les traits définitoires du fait religieux et les mutations intervenues dans l'institution politique et les politiques publiques et sociales au cours de cette "modernité » et plus particulièrement, après 1945. Cette articulation est structurante et fortement directrice dans la mise en œuvre d'un programme de recherche, dès lors que celui-ci s'inscrit dans une réflexion, d'une portée éminemment publique et politique, sur «Welfare and Religion». Or si le livre fait jouer un rôle heuristique, guère discutable, au binôme "believing and belonging ", et si la contribution d'Anders Bäckström intitulé «Welfare, Society and Secularization » replace utilement au cœur de ce livre une réflexion sur les politiques publiques de providence, welfare, et les mutations successives dans les demandes adressées par les sujets sociaux aux institutions en charge du religieux, il manque à ce volume une interrogation d'ordre plus général sur les "mutations » invoquées dans le titre, qui ont conduit, notamment au cours des dernières décennies, à l'épuisement des politiques publiques de l'État-providence, dont l'apogée après 1945 a pu sembler le concomitant de l'avènement d'une société inclusive et sécularisée. En effet, lorsqu'Anne Brigitta Pessi conclut (p. 203) que «les actions et les paroles d'altruisme et de justice de la part des institutions religieuses sont perçues comme un contrepoids aux rudes valeurs de concurrence et à l'injustice qui en découle ", "The acts and words of altruism and justice by religious institutions are seen as a countervailing force against the harsh values of competition, and the resulting injustice», on peut constater et regretter l'absence d'un cadre plus général servant à explorer le tournant du "post-sécularisme ». Car si le concomitant de la réémergence du religieux est l'épuisement d'un paradigme séculariste qui légitimait la mise en place des institutions d'une providence sécularisée, et si cette réémergence est désormais le préalable d'un nouvel agencement fondé sur l'articulation entre "Welfare » et "Religion", il faudrait prendre la mesure de l'efficace ruse de l'histoire qui aura fait du religieux l'adjuvant utile d'une réaffirmation des « rudes valeurs de concurrence et de l'injustice qui en découle».

4 Ce livre laisse donc en suspens des interrogations qu'elle amorce cependant, concernant les conditions de possibilité d'un "sécularisme", qui serait désormais révolu, et les conditions de possibilité de cette révolution, dont les effets sont sociaux et culturels, entraînant une nouvelle visibilité de la religion, à rebours de sa privatisation et de sa personnalisation. Notons que ces effets sont aussi plus trivialement universitaires : en témoigne la fierté perceptible dans le constat d'Anne Birgitta Pessi (p. 211) qui clôt ce volume : "large numbers of researchers from various disciplines are currently engaged in the study of religion. More particularly, they are finally inviting and including us, scholars of religion, to work together in cross-disciplinary networks. As Grace beautifully has put it $(20146,125)$ Cinderella has finally been invited to the ball ». Ce volume recenserait donc les pas de danse de Cendrillon, enfin réadmise au bal des sciences sociales. Insistons sur l'intérêt de ce volume, au moment de la visibilité retrouvée du fait religieux, moment par ailleurs marqué par les tensions ou fractures qui affectent le projet européen et qui est aussi celui de la longue "provincialisation " (Chakrabarty, 2000) de l'Europe. Ce livre fait réfléchir, par les convictions qui portent le propos des auteurs, par ses impensés, par sa focalisation exclusive sur les 
manifestations nord-européennes du fait religieux. Ce repli conditionne sa cohérence et sa conviction. Manque cependant à ce travail la réflexivité critique par laquelle une entreprise de recherche fait retour sur son objet ou terrain, sa méthode et ses conclusions.

Car s'il y a bien un domaine des sciences sociales où la notion de neutralité méthodologique ne va pas de soi, où toute approche est nécessairement située, conjoncturellement et culturellement circonscrite, c'est bien celui du fait religieux, " the study of religion ", pour citer le titre de ce livre. Or passée la première contribution, "Religion as A Grammar of Memory: Reflections on a Comparison between Britain and France " de Danièle Hervieu-Léger, les différentes contributions attestent les affinités d'approche et d'objet entre la Grande-Bretagne et les pays scandinaves. Cela est relevé, comme une évidence et sans réserve, par Abby Day lorsqu'elle remarque dans une note, à propos de l'articulation entre traits d'appartenance (belonging) et de croyance (believing) étudiés par Grace Davie dans le contexte du Royaume-Uni, que « des schémas similaires se retrouvent dans d'autres pays "nordiques", à telle enseigne que le constat l'aura incité à émettre l'hypothèse, avec Mia Lövheim, Grace Davie et d'autres collègues, que nous formions un réseau informel de chercheurs qui étudient la religion dans l'Europe du Nord et au Canada - des pays protestants dont l'héritage historique est d'avoir eu une église nationale et dont la population actuelle a pour caractéristique d'être nominalement chrétienne » (p. 207).

Une thèse générale sous-tend les différentes contributions à ce volume : «la relation entre la religion et le séculier ne s'est pas déroulée comme prévu [...] il existe un plus grand pluralisme religieux et [que] les formes de la croyance religieuse se sont déplacées, de l'institutionnel au personnel " (Dinham, p. 53). Ces constats s'appuient sur les travaux de Grace Davie et, en amont, sur ceux de David Martin, qui dans sa contribution au volume énonce que «la modernisation, comprise comme rationalisation, n'a jamais été ni une voie unique, avec le nord-ouest de l'Europe en position de pionnier, ni une double voie, où la France et la Suède auraient tracé les deux options globalement disponibles» (p. 86). Le constat de la persistance du fait religieux en Europe, précisément à travers la période où la "secular theory " était à son apogée (Dinham), forme la trame de ce livre. Il fonde la préconisation d'une approche moins catégorique, davantage attentive aux contextes spécifiques, pour l'étude du fait religieux et de son interaction avec le processus de la «modernité ». Mais la réserve que l'on peut formuler vis-à-vis de l'approche adoptée, y compris lorsqu'il s'agit de pointer les signes manifestes de cette persistance du fait religieux, tient à une propension à faire de la sous-explicitation des catégories un avantage argumentatif: récusation des catégories générales de modernité et de sécularisation, adoption du pluriel, modernities, constat d'une persistance avérée du religieux, mais à travers des procédures qui à leur tour ont tendance à produire les données qui pourront valider la justesse des catégories employées; articulation accommodante entre believing et belonging (Abby Day, p. 95-111), en une variation sur l'antithèse entre ces mêmes termes qui fut posée par Grace Davie à un stade antérieur de sa recherche; usage de la notion de "vicarious religion" (Davie, 2007), "religion par procuration", pour qualifier une situation où une majorité de non participants délèguent à une minorité active la charge de faire perdurer la structure institutionnelle à l'égard de laquelle on signale un consentement implicite. "Vicarious religion » n'est nullement la catégorie-charnière d'un grand récit. Typique en cela du mode d'argumentation développé dans le livre, la catégorie requiert, pour que sa pertinence puisse être constatée, une identification 
minimale et tacite de la part de ceux qui agissent "par procuration ». Si donc le grand récit de la sécularisation s'est épuisé en raison de son finalisme téléologique présomptueux, rationnel et progressif, le récit en mineur que porte la recherche collaborative de ce livre s'auto-valide à travers le constat aisé de faits aussi accommodants que ceux qui sont corrélatifs de «vicarious religion» ou des variations sur le binôme «believing and belonging ".

Manque donc une réflexion sur les conditions culturelles qui font de ce nord-ouest européen le terrain propice à la validation des hypothèses qui sont avancées ici. Même un Festschrift aurait besoin de quelques grains de sel (et le recours omniprésent dans ce volume au prénom "Grace » pour désigner la chercheuse Grace Davie, dont on veut interroger et prolonger la recherche, n'autorise pas l'interrogation critique des thèses). La lecture de ce livre appelle donc une ouverture vers des terrains ailleurs en Europe et dans le monde, afin d'inventorier d'autres versions du moment post-séculier, pour ainsi donner plus de substance au projet que porte ce livre, qui est d'explorer les diverses modalités d'une condition post-séculière, qui serait le stade actuel d'une modernité plurielle, désormais polyarchique. Or une telle polyarchie ne relève pas nécessairement de la grammaire ordonnée qui est exposée ici, qui vaut sans doute pour le Nord-ouest européen, grammaire faite d'articulations ajustées entre croyance et appartenance, d'heureuse collaboration entre un accomplissement direct et minoritaire d'une communauté de destin religieux et un accomplissement consenti, par procuration, de la part d'une majorité. La remarque de David Martin, qui seul parmi les participants émet quelques réserves, mérite l'attention: «Even-handedness is not fairness. Sometimes truth may be extreme, dark and paradoxical» (p. 82). La persistance du fait religieux, dont le constat sert d'amorce au projet de recherche de Grace Davie et des contributeurs à ce volume, appelle plus que le balancement équilibré entre "believing " et "belonging ", sauf à faire de la sociologie de la religion, telle qu'elle est mise en œuvre à partir des données issues d'une certaine exploration du terrain du Nord-ouest européen, une entreprise strictement idéographique, dont le pouvoir descriptif se limite à son aire de production et de réception collégiale. 